\title{
FORWARD AND BACKWARD STIMULATED RAMAN \\ SCATTERING IN A MULTIMODE FIBER*
}

\author{
C.-H. Lin, M.A. Nelson, B.R. Marsha11, and J.K. Theobald \\ EGEG, Inc., Santa Barbara Operations \\ 130 Robin Hil1 Road, Goleta, California 93017
}

\section{ABSTRACT}

Several orders of forward and backward stimulated Raman scattering are generated by a pulsed dye laser in a multimode step-index fiber. Frequency spectra and temporal profiles at different power levels are recorded and discussed.

*This work was performed under the auspices of the U.S. Department of Energy. NOTE: By acceptance of this article, the publisher and/or recipient acknowledges the U.S. Government's right to retain a nonexclusive royalty-free license in and to any copyright covering this paper. 


\section{DISCLAIMER}

This report was prepared as an account of work sponsored by an agency of the United States Government. Neither the United States Government nor any agency Thereof, nor any of their employees, makes any warranty, express or implied, or assumes any legal liability or responsibility for the accuracy, completeness, or usefulness of any information, apparatus, product, or process disclosed, or represents that its use would not infringe privately owned rights. Reference herein to any specific commercial product, process, or service by trade name, trademark, manufacturer, or otherwise does not necessarily constitute or imply its endorsement, recommendation, or favoring by the United States Government or any agency thereof. The views and opinions of authors expressed herein do not necessarily state or reflect those of the United States Government or any agency thereof. 


\section{DISCLAIMER}

Portions of this document may be illegible in electronic image products. Images are produced from the best available original document. 
FORWARD AND BACKWARD STIMULATED RAMAN

SCATTERING IN A MULTIMODE FIBER*

\section{C.-H. Lin, M.A. Nelson, B.R. Marshall, and J.K. Theobald EGEGG, Inc. , Santa Barbara Operations \\ 130 Robin Hill Road, Goleta, California 93017}

\section{INTRODUCTION}

Forward stimulated Raman scattering, ${ }^{1}$ backward Raman amplification, ${ }^{2}$ and continuous-wave Raman oscillation ${ }^{3}$ in single mode fibers have been reported previously. Forward stimulated Raman scattering (SRS) in multimode fibers has also been observed. ${ }^{45}$ In a previous paper we reported the observation of backward SRS in a multimode fiber. ${ }^{6}$ In this report, we discuss the temporal evolution of various orders of forward and backward stimulated Raman scattering generated in a long multimode fiber by a pulsed laser. The long fiber length and high pump power available allow different orders of forward Stokes emission to develop in different regions of the fiber which, in turn, generate next higher orders of backward emission. Further, a long pulse duration permits them to be substantially amplified.

\section{EXPERIMENT}

The experimental arrangement is shown in Fig. 1. The light shource is a flashlamp pumped dye laser operated at $594 \mathrm{~nm}$ with a linewidth of $0.6 \mathrm{~nm}$. An aperture is placed in the laser cavity to select the $\mathrm{TEM}_{\mathrm{O}}$ mode and to limit the beam size at the exit window to $2.5 \mathrm{~mm}$. The pulse width varies from 1.6 to $2 \mu \mathrm{sec}$ depending on the flashlamp pump power. The multimode step-index fiber used in

*This work was performed under the auspices of the U.S. Department of Energy. NOTE: By acceptance of this article, the publisher and/or recipient acknowledges the U.S. Government's right to retain a nonexclusive royalty-free license in and to any copyright covering this paper. 
this experiment is one of seven in a Corning Corguide cable. Its core diameter is $85 \mu \mathrm{m}$, the numerical aperture is 0.175 , the attenuation constant is $12 \mathrm{db} / \mathrm{km}$ at $594 \mathrm{~nm}$, and the fiber length as measured by an optical time domain reflectometer is $510 \mathrm{~m}$.

The laser beam is focused into the fiber by a $2-\mathrm{cm}$ focal length lens. The forward stimulated Raman emission and the residue pump beam exiting from the fiber is directed into the slit of an optical multichannel analyzer (OMA) which measures their spectrum. ${ }^{7}$ The backward waves emitting from the input end of the fiber and the portion of the pump beam reflecting from the input surface are also directed by mirrors into the OMA slit, but are spatially separated from the forward emissions. The programming capability of the OMA allows the spectra of the forward and backward waves to be displayed simultaneously and separately. A portion of the forward emission is Fresnel-reflected from the fiber exit surface back through the fiber. These data may also be recorded with the backward emission, but are small by comparison.

The emissions are directed into a monochromator, enployed as a spectrometer by removing the exit slit, for measuring the time history of all orders of backward SRS and the surface reflected pump beam. " Scanning the monochromator spatially separates and directs different orders of stimulated emission to either of two photodiode detectors, $\mathrm{PD}_{1}$ and $\mathrm{PD}_{2}$. $\mathrm{PD}_{1}$ detects the temporal shape of the pump pulse, and lower order emissions. $\mathrm{PD}_{2}$ detects the time history of higher order emissions or, by using the stopper $S$, detects only a specific order of Stokes emission. The signals from $\mathrm{PD}_{1}$ and $\mathrm{PD}_{2}$ are recorded on a dual beam oscilloscope with synchronized time sweep. For recording the time history of the forward emission, outputs from the exit end of the fiber are directed into the monochromator and similarly treated.

We determine the power coupled into the fiber in the conventional way by tapping off a portion of the laser pump beam into a radiometer, using a beam splitter of known reflectivity. After the experimental measurements are completed, the fiber is cut near the input end and, without altering the coupling condition, the energy transmitted through the short piece is measured. From the ratio of these readings the efficiency of coupling light into the fiber, including loss due to Fresnel reflection at the lens and the fiber end, is shown to be typically about $60 \%$. The peak pump power is determined from the injected energy measurement and the time profile of the input laser pulse recorded on the oscilloscope. 


\section{RESULTS}

First-order forward Stokes emission centered at $611 \mathrm{~nm}$ was observed at a peak pump power of $1.3 \mathrm{~kW}$. Temporal profiles of first-order emission at several peak pump power levels are shown in the lower trace of each photo in Fig. 2. Top traces show the residue of the 594-nm pump beam. The small periodic noise on the tail of the pulses is electrical noise from the laser trigger system. Calibrations for the pulse intensity are, respectively, $1.4 \mathrm{~kW} / \mathrm{V}$ for the pump beam and $0.95 \mathrm{~kW} / \mathrm{V}$ for the Stokes emission, except for Fig. 2a, which should be multiplied by a factor of eight.

-As the pump power increases from the SRS threshold value, the Stokes pulse width first reduces to a minimum of $400 \mathrm{~ns}$. Then, as the pump power rises over - $2.5 \mathrm{~kW}$, the Stokes pulse starts to broaden and finally converts to the next-order emission, severely depleting the pump pulse in the process. The hole in the lower trace of Fig. 2d indicates that most of the first-order Stokes emission has converted to higher-order emissions. Also, note that the maximum power of the residue pump pulse emitted from the fiber is roughly independent of the intensity of the pump input and is approximately equal to $400 \mathrm{~W}$.

The time history of each of six forward Stokes emission, all generated by similar pump pulses of approximately the same power, are shown in the lower traces of Fig. 3a through 3f. The upper traces show the forward pump pulse residual and all forward orders lower than that displayed in the lower trace. The peak pump input was about $16 \mathrm{~kW}$. A typical frequency spectrum of the total pulse emission. at this power is shown in Fig. $3 \mathrm{~g}$, recorded concurrently with trace $3 f$. Trace $3 \mathrm{~h}$ is a high resolution trace of sixth-order emission showing time history detail obtained using a wideband oscilloscope. Similar measurements have been made at several other pump power levels.

Several observations can be made about the results.

The maximum output power of the forward Stokes emissions are relatively constant at $0.95,1.34,1.56$, and $1.88 \mathrm{~kW}$, respectively, for first-, second-, third-, and fourth-order, despite large variation in the pump power.

Small amplitude variations in the temporal profile of the pump pulse are amplified to much larger fluctuations in high-order forward Stokes pulses. This is most vividly shown in Fig. 3h, the sixth-order emission detected by a fast oscilloscope. It consists of several spikes of less than 20 ns duration each. 
Because of this amplified amplitude fluctuation, the middle portion of the high-order forward Stokes pulses are not completely converted to higher-order emissions, such as is exhibited by the pump pulse and the low-order Stokes pulses.

Considerable amounts of laser pump pulse energy have been converted to backward wave. This is illustrated more clearly in Fig. 4, which displays the summation of all forward Stokes pulses and the residue pump beam at two input power levels. Fig. 4a, lower trace, results from an unattenuated pump input; Fig. $4 b$ shows the output with a similar input pump pulse, except that it was attenuated by a factor of 20 before it passed through the fiber and did not generate any stimulated Raman scattering.

Each temporal component of forward Stokes pulse is amplified by the corresponding temporal component of the generating pump pulse. This limits the maximum Stokes amplitude and causes fast intensity fluctuations. The backward Stokes pulse on the other hand, is amplified over the longer time segment of pump pulse it traverses in the reverse direction, resulting in gain that is proportional to the total energy of that segment of pump pulse. It may have, in fact, a peak intensity exceeding the pump intensity, with a smooth, bell-like pulse shape.

First-order backward Stokes emission was observed at a peak pump power of $1.8 \mathrm{~kW}$. The lower traces of Fig. 5 show the shape of first-order 611-nm backward Stokes pulse at increasing pump powers. The upper traces are the portion of pump pulse reflected from the entrace surface of the fiber. The backward Stokes pulse is very broad near threshold. (The second pulse in the lower trace of Fig. 5a is the forward Stokes pulse reflected from the exit surface of the fiber.) Unlike forward Stokes emission the gain of the backward Stokes wave does not saturate; as the pump power increases, the backward wave amplitude also increases and its width decreases. For example, with a pump power increase to $5 \mathrm{~kW}$ the backward pulse width decreases from its threshold value to $300 \mathrm{~ns}$. Further increasing the pump power to $11 \mathrm{~kW}$ decreases the backward pulse width to $200 \mathrm{~ns}$ and increases its peak amplitude by a factor of 3 .

However, gain is not linearly proportional to pump power, as illustrated in Fig. 5 by the much smaller increase in peak Stokes pulse intensity from a pump power of $3.2 \mathrm{~kW}$ to $5.0 \mathrm{~kW}$, compared to that from $2.3 \mathrm{~kW}$ to $3.2 \mathrm{~kW}$. The reduction in amplification factor is due not only to the depletion of the pump power by the forward stimulated emission, but also to the earlier initiation of the backward wave at higher pump power. This forward time shift of the Stokes pulse is indicated 
by the reduced interval between peaks of the pump pulse and the Stokes pulse, from $800 \mathrm{~ns}$ at $3.2 \mathrm{~kW}$ to $500 \mathrm{~ns}$ at $5.0 \mathrm{~kW}$. Whether this occurs because the Stokes pulse originates from the leading edge of the pump pulse or because it is initiated closer to the entrance end of the fiber, the net effect is to reduce the total pump pulse energy that is traversed by the Stokes pulse.

The time profile of each of the next three orders of backward Stokes emissions are shown in the lower traces of Fig. 6, generated at pump powers of $7.7,10.7$, and $15 \mathrm{~kW}$, respectively. The second pulses in each case are, again, forward Stokes emission of the same order reflected back from the exit end of the fiber. The upper traces show the sum of all lower-order Stokes emissions than the lower trace pulse, plus the reflection of the pump beam from the entrance surface. Each of the first three orders of backward emission are shown in succession in the lower traces of Fig. 7a, b, and c, at a peak pump power of $13 \mathrm{~kW}$. The sum of all three orders are shown in the lower trace of Fig. 7d. Top traces again show all lower orders than the lower trace display, plus the reflected pump beam. These and other results lead to several interesting observations.

Because each order of backward Stokes emission is generated by the preceding order of forward emission, and because higher-order forward stokes emissions are generated further from the entrance end of the fiber, different orders of backward Stokes pulses can coexist (in contrast to the forward emission), and higher-order Stokes pulses always lag behind the lower-order ones.

Temporal pulse profiles localize, approximately, the initiation of the backward Stokes pulse. For example, assuming the peak of the Stokes pulse in Fig. 5a originates from the peak of the pump pulse, it can be estimated from the time between their peaks that this pulse is initiated at a point in the fiber roughly $100 \mathrm{~m}$ from the entrance surface. In Fig. 6c, assuming that the peak of the fourth-order backward Stokes pulse is originated from the peak of the thirdorder forward Stokes pulse, it can be estimated that the fourth-order pulse is initiated roughly $300 \mathrm{~m}$ from the entrance end of the fiber.

Backward Stokes pulses initiate from a region increasingly closer to the input end of the fiber as the pump power increases. For instance, the third-order Stokes pulse is initiated from a point about $250 \mathrm{~m}$ from the fiber input end at a pump power of $10.5 \mathrm{~kW}$, as compared to about $140 \mathrm{~m}$ from the input at $13 \mathrm{~kW}$. These distances also roughly indicate where second-order forward Stokes pulses begin to saturate, at the corresponding input powers. 
The data of Fig. 7a show that the first-order Stokes pulse originates from the leading portion of the pump pulse, and at a point close to the entrance surface. of the fiber. This allows smaller pulses of the same order to develop from later portions of the pump pulse. They appear, for instance, at $0.9 \mu \mathrm{s}$ and $1.8 \mu$ s later than the first pulse. The second-order backward Stokes pulse is also trailed by a forming small pulse of its same ordẹ at $1.8 \mu \mathrm{s}$ later.

This work was funded by EGGG's internally directed R\&D program through the

- DOE's Nevada Operations Office under Contract No. EY-76-C-08-1183, to determine the maximum linear power capability of an optical fiber in a large bandwidth, analog nuclear data transmission system. 


\section{REFERENCES}

1. R.H. Stolen, E.P. Ippen, and T.R. Tynes, AppZ. Phys. Lett., 20, 62. (1972).

- 2. Chinlon Lin and R.H. Stolen, Appl. Phys. Lett., 29, 428 (1976).

3. K.0. Hil1, B.S. Kawasaki, and D.C. Johnson, Appl. Phys. Lett., 29, 181 (1976).

4. S.M. Jensen and M.K. Barnoski, in Technical Digest of Topical Meeting on Optical Fiber Trausmission II, Paper No. 'l'uD7-1 (February 1977).

5. J. Botineau, F. Gires, A. Sä̈ssy, C. Vanneste, and A. Azema, App Z. Opt., 17, 1208 (1978).

6. C-H Lin, B. Marshall, M. Nelson, and K. Theobald, to be published in Applied Optics.

7. Optical Multichanne1 Analyzer OMA-2 is manufactured by Princeton Applied Research Corporation, Princeton, New Jersey. 
FIGURE CAPTIONS

Figure 1 - Experimental arrangement.

- Figure 2 - First-order forward stimulated Raman scattering. Bottom trace in each photo is the first-order Stokes emission centered at $611 \mathrm{~nm}$. Top trace is, the residue pump pulse. Peak powers of the injected pump pulse are a) $1.4 \mathrm{~kW}$, b) $2.1 \mathrm{~kW}$, c) $3.6 \mathrm{~kW}$, and d) $5.3 \mathrm{~kW}$.

Figure 3 - First six orders of forward Stokes emission at a pump power of approximately $16 \mathrm{~kW}$. Lower traces of a) through f) are first-.through sixthorder Stokes emissions. Wavelengths are centered, respectively, at $611,628,645,664,685$, and $706 \mathrm{~nm}$. Upper traces are sums of the residue pump pulse and all orders of Stokes emissions lower than the one shown in the lower trace. Peak pump powers are a) $16.3 \mathrm{~kW}$, b) $16.3 \mathrm{~kW}$, c) $16.5 \mathrm{~kW}$, d) $15.9 \mathrm{~kW}$, e) $16.1 \mathrm{~kW}$, and f) $16.0 \mathrm{~kW}$. Photo g) is the frequency spectrum taken concurrently with pulse f). The left hand side displays the spectrum of forward emission and the right hand side that of backward emission. Photo $h$ ) is the sixth-order Stokes emission recorded on a wideband Tektronix 519 oscilloscope at a peak pump power of $16.0 \mathrm{~kW}$.

Figure 4 - Comparison of the pump pulse and the sum of forward emissions. Pulse a) is the sum of the residue pump pulse and all forward Stokes emissions, at a peak pump power of $16 \mathrm{~kW}$. Pulse b) is a similar display except that the pump pulse is attenuated by a factor of 20 before passing through the fiber.

Figure 5 - First-order backward Stokes emission. Lower trace in each photo is the first-order Stokes emission. Upper trace is the portion of pump pulse reflected back from the entrance surface of the fiber. Second pulse in the lower trace of a) is the first-order forward Stokes pulse reflected back from the exit surface of the fiber. Peak pump powers are a) $2.3 \mathrm{~kW}$, b) $3.2 \mathrm{~kW}$, and c) $5.0 \mathrm{~kW}$. 
Figure 6 - Second-, third-, and fourth-order backward Stokes emissions. First. pulses in lower trace of a), b), and c) are, respectively, second-, third, and fourth-order backward Stokes emissions. Second pulses are exit end reflections of forward emissions of the same order. Upper trace in each photo is the sum of all orders of Stokes emission lower than the one specified, plus the reflection of the pump pulse. Peak pump powers are a) $7.7 \mathrm{~kW}$, b) $10.7 \mathrm{~kW}$, and c) $15 \mathrm{~kW}$.

Figure 7 - First three orders of backward Stokes emission at peak pump powers of approximately $13 \mathrm{~kW}$. Lower traces are a) first-, b) second-, c) third-, and d) first-, second-, and third-order Stokes emission. Upper trace in each photo is the surn of all orders of Stokes emission lower than that specified, plus the reflection of the pump pulse. Peak pump powers are a) $12.9 \mathrm{~kW}$, b) $13.3 \mathrm{~kW}$, c) $13.3 \mathrm{~kW}$, and d) $13.0 \mathrm{~kW}$. 


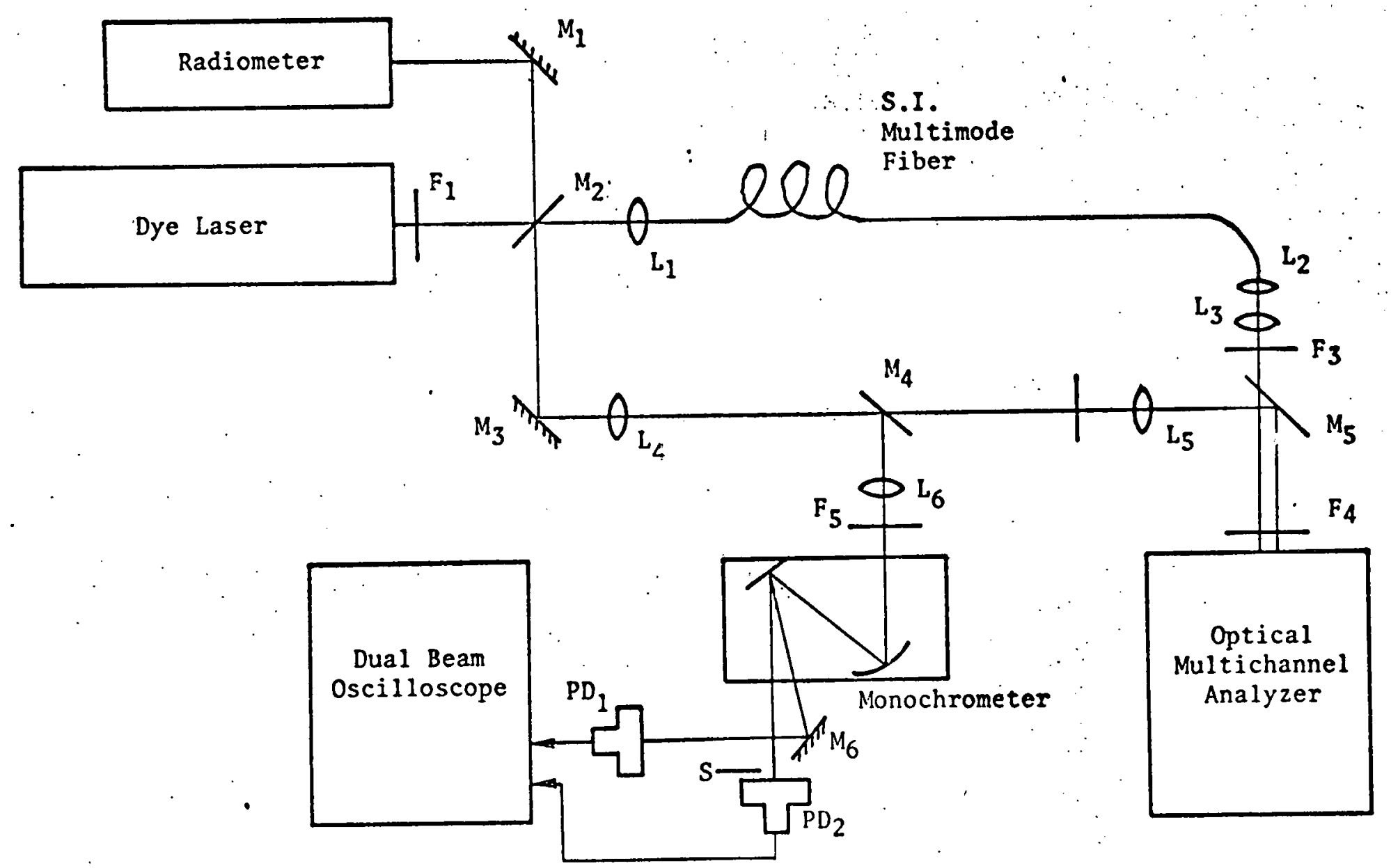

Figure 1 


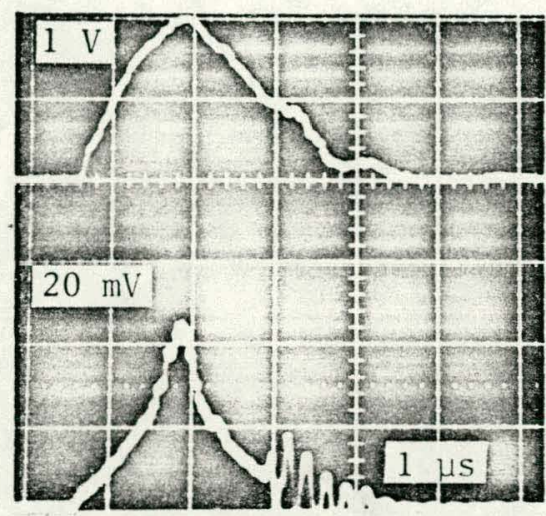

(a)

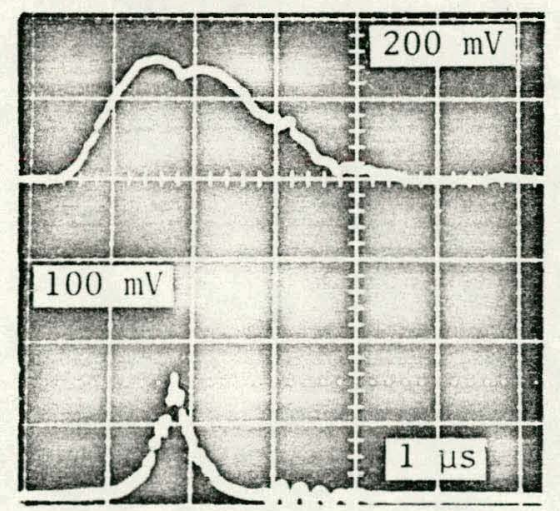

(b)

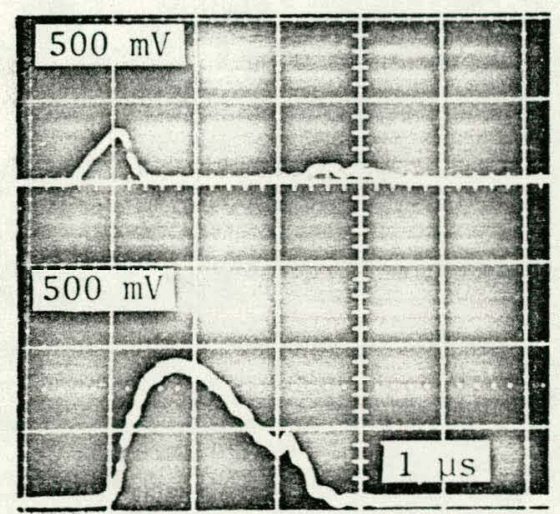

(c)

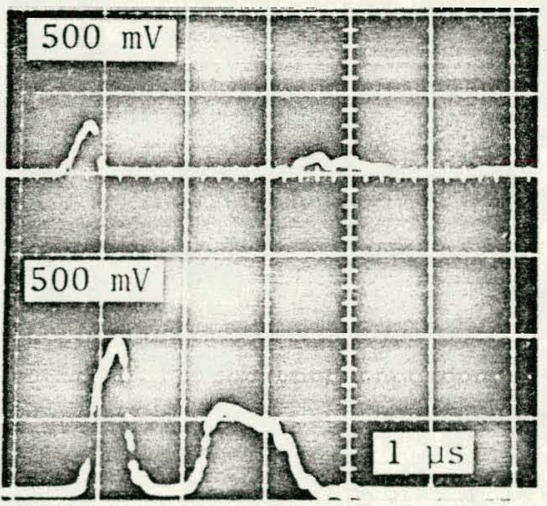

(d) 


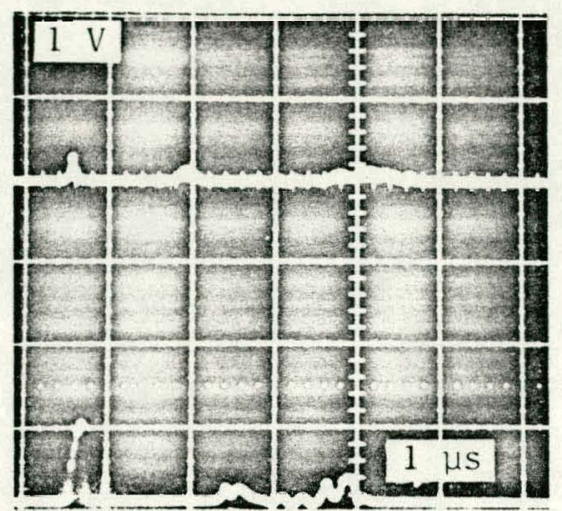

(a)

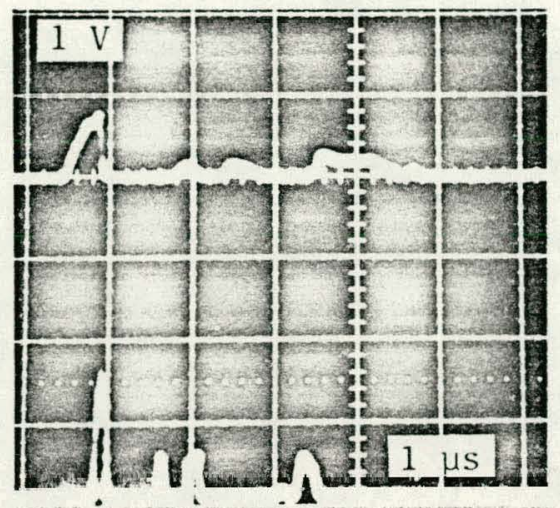

(c)

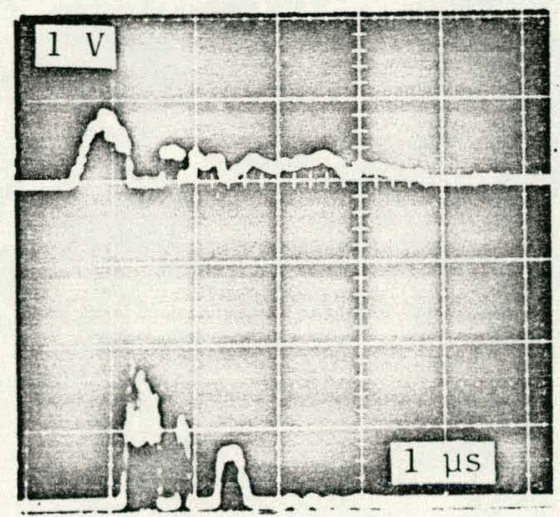

(e)

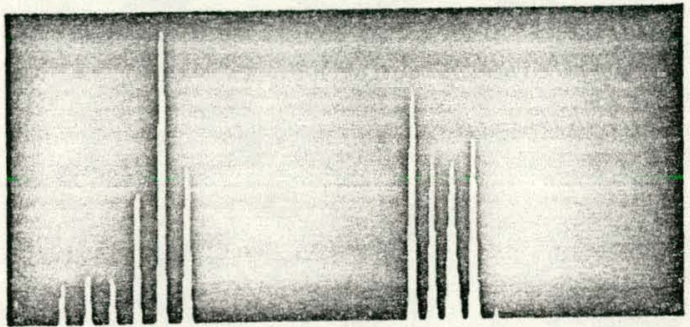

(g)

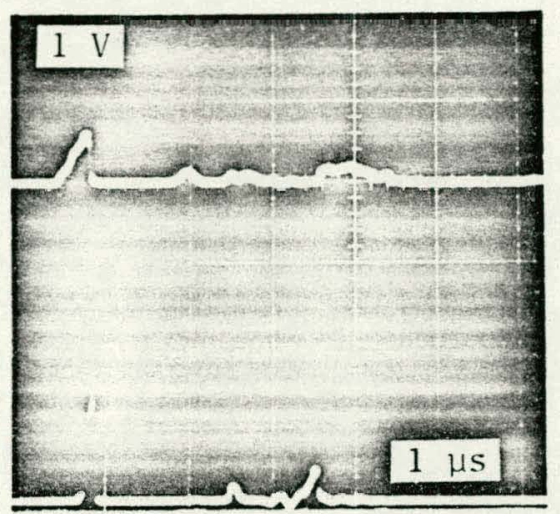

(b)
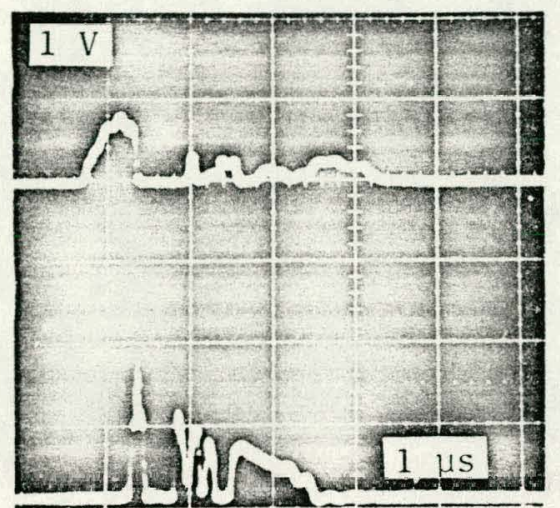

(d)

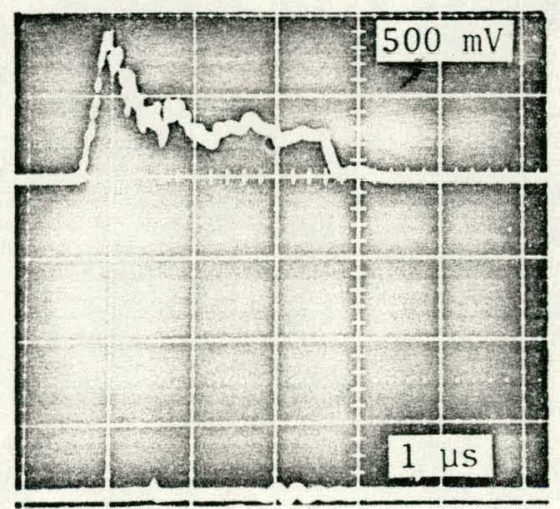

(f)

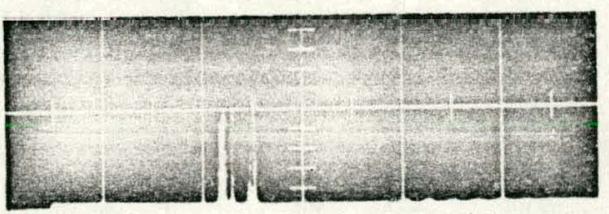

(h) 


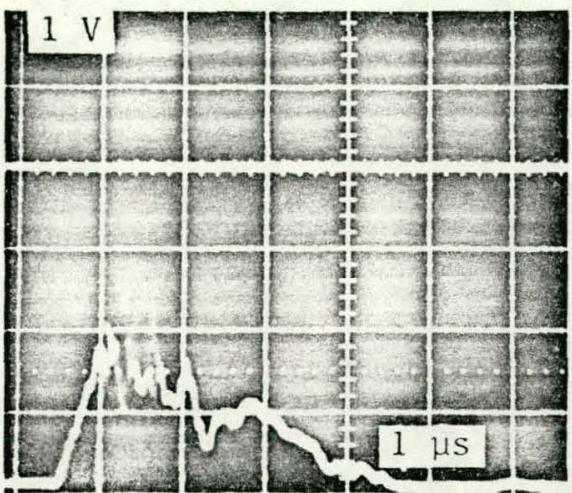

(a)

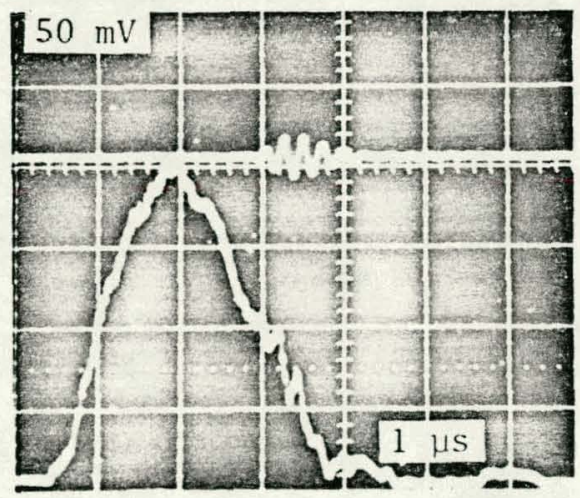

(b) 


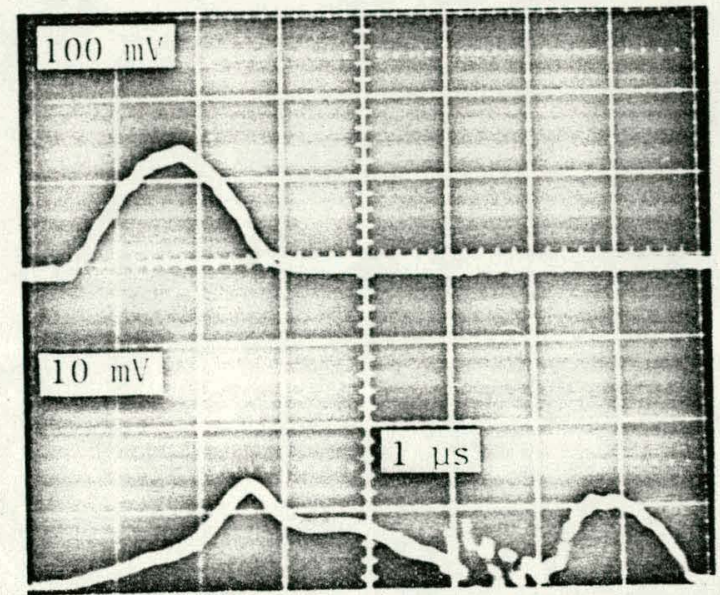

(a)

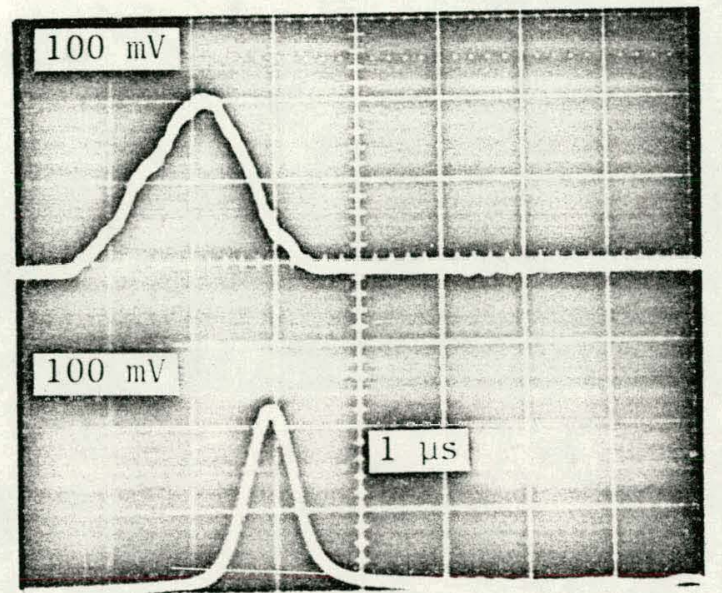

(b)

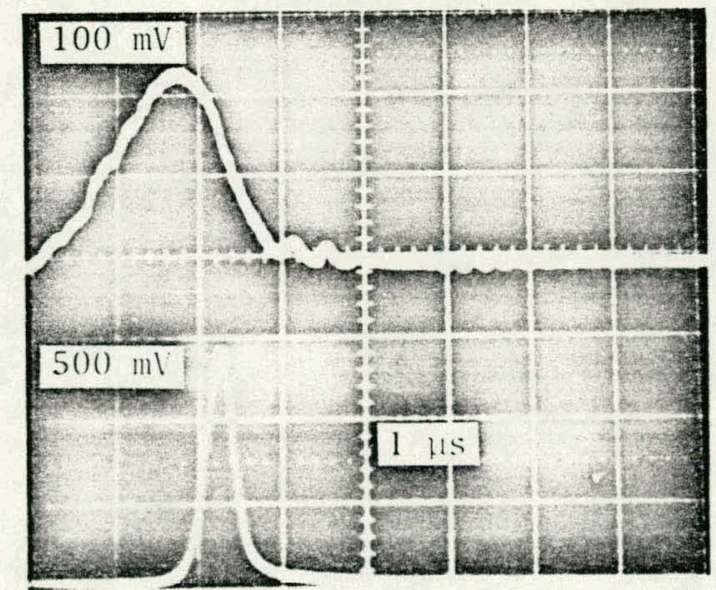

(c) 

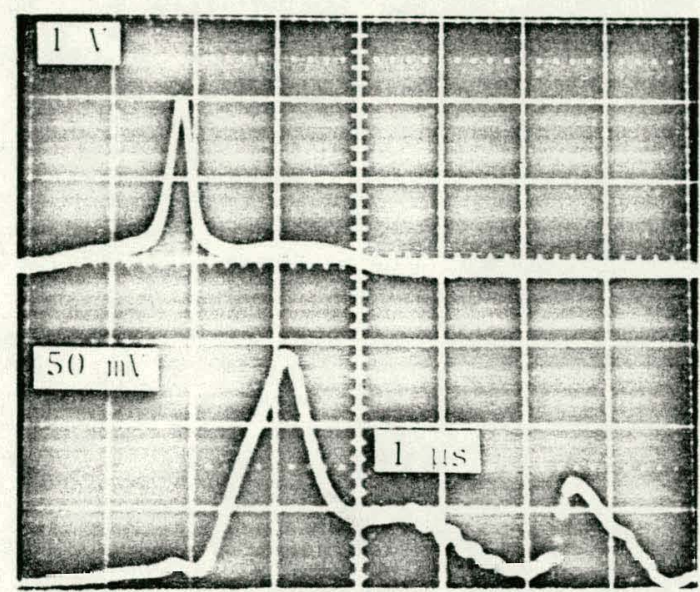

(a)

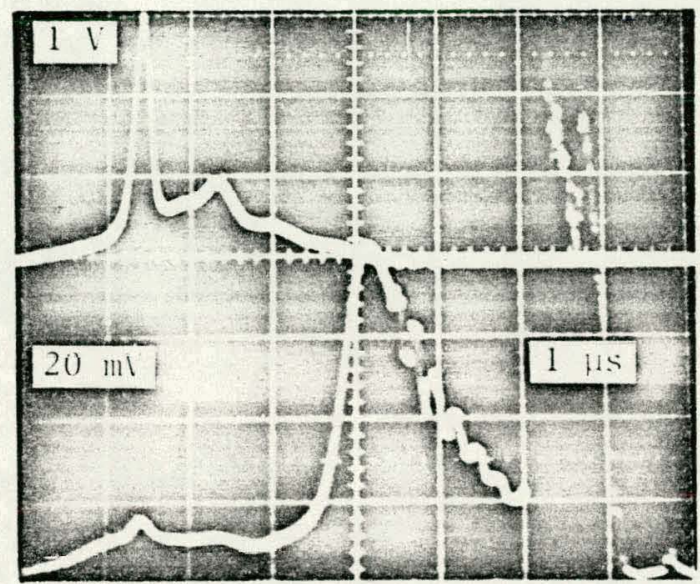

(b)

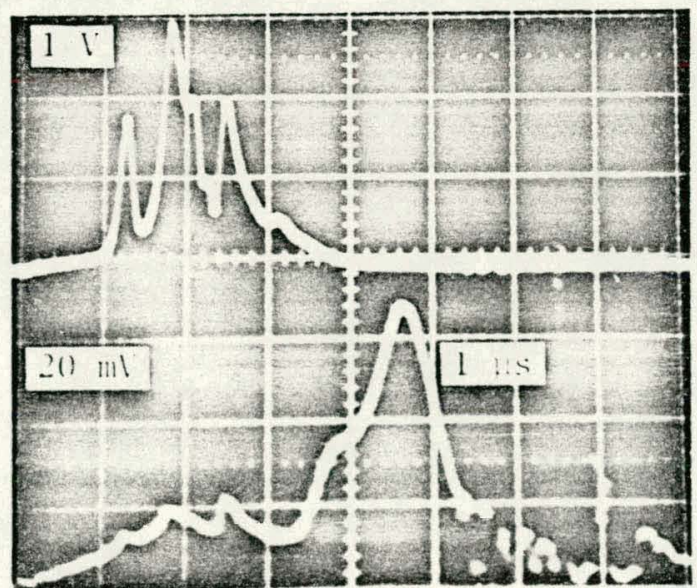

(i) 


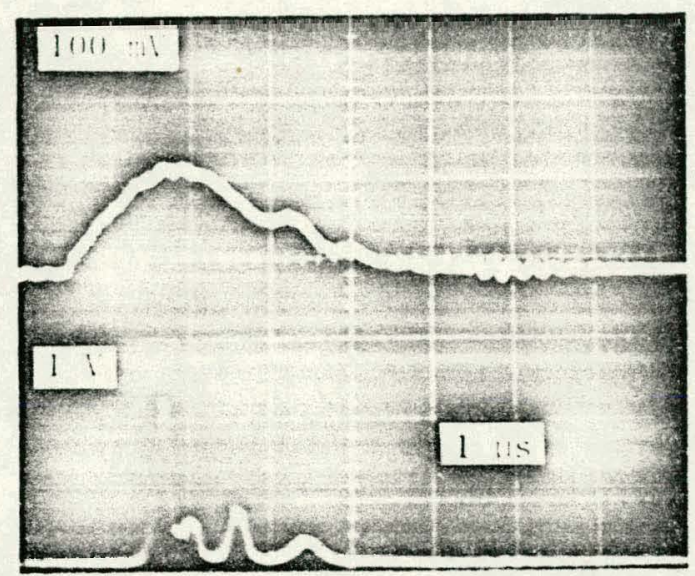

(a)

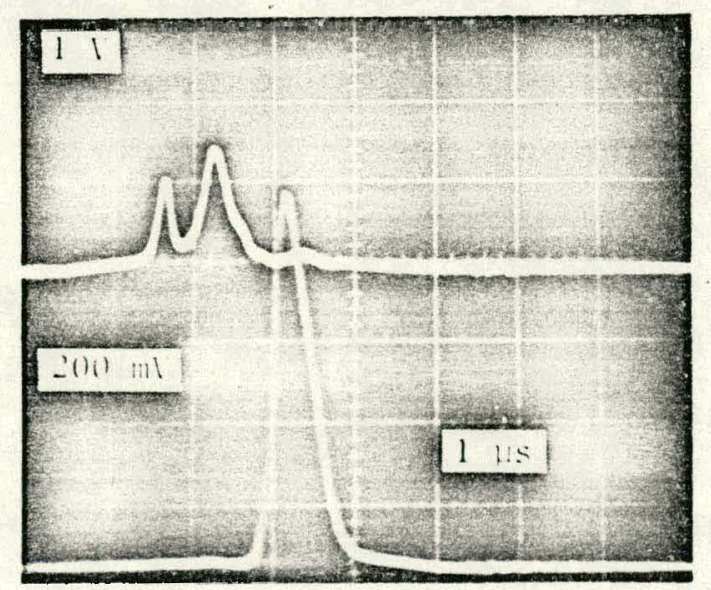

(c)

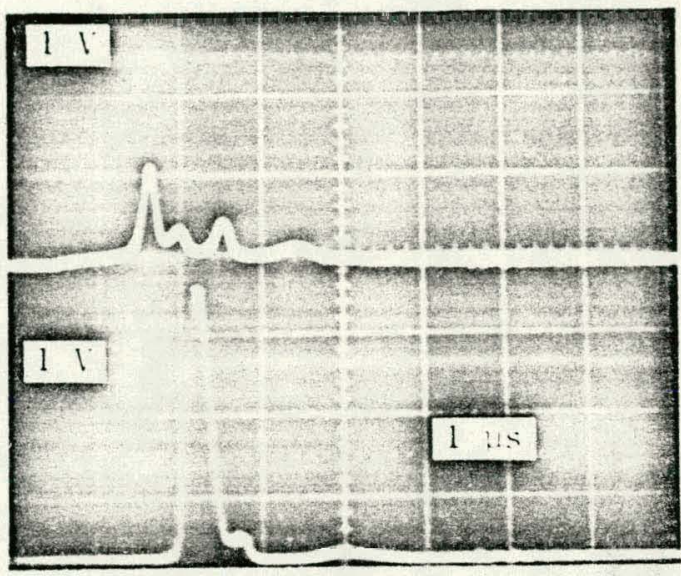

(b)

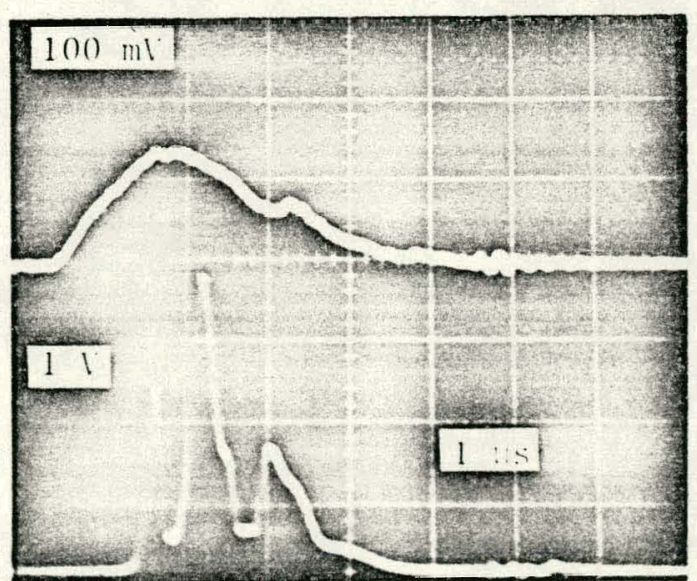

(d) 